\title{
Phenytoin Induced Truncal Ataxia: Need For TDM
}

\author{
Anup jagarlamudi*, Asma Begum, Nazmeen Saba, Mahalakshmi Teegala \\ Department of Clinical Pharmacy Practice, Pullareddy Institute of Pharmacy, Hyderabad, Telangana, INDIA.
}

\begin{abstract}
Phenytoin is a hydantoin derivative with a narrow therapeutic index and is indicated in GTCS, nonepileptic seizures, status epilepticus, neuritic pain, as skeletal muscle relaxant, ventricular arrhythmias unresponsive to lidocaine or procainamide, arrhythmias induced by cardiac glycosides, and in prophylactic control of seizures during neurosurgery. The usual practice is that phenytoin is given along with other anticonvulsant drugs like benzodiazepines or valproic acid in case of seizures. But this combination of antiepileptic drugs have got serious drug-drug interactions which usually results in exaggerated effects of either of the drugs. A female patient of 24 years admitted with seizures was put on phenytoin, lorazepam, and valproic acid. After a few days of therapy, she had 2 more episodes of seizures and later on developed nystagmus and truncal ataxia. Even though the effects were attributed to "drug", the physicians failed to take necessary action. Lack of a protocol for monitoring plasma concentrations of the narrow therapeutic index drug was responsible for the toxic effects of Phenytoin and by conducting TDM for phenytoin, such toxic effects could have been avoided.
\end{abstract}

Key words: Phenytoin, Narrow therapeutic index, Seizures, Truncal ataxia, Nystagmus, Plasma concentrations.

\section{INTRODUCTION}

Phenytoin is a hydantoin derivative and is classified as an anticonvulsant. Phenytoin is indicated in generalised tonic-clonic seizures, status epilepticus, nonepileptic seizures (post-head trauma, Reye's syndrome), neuritic pain (migraine, trigeminal neuralgia, and Bell's palsy), as a skeletal muscle relaxant, in ventricular arrhythmias unresponsive to lidocaine or procainamide, and arrhythmias induced by cardiac glycosides, and for prophylactic control of seizures during neurosurgery. ${ }^{1}$ Phenytoin is a narrow therapeutic index drug and its serum concentrations has to monitored throughout the therapy.

The therapeutic plasma levels of phenytoin are $10-20 \mathrm{mcg} / \mathrm{ml}$, although in some patients, they occur at 5 to $10 \mathrm{mcg} / \mathrm{ml}^{1}$

The adverse reactions of phenytoin are: ataxia, slurred speech, dizziness, insomnia, nervousness, twitching, headache, mental confusion, decreased coordination, hypotension, nystagmus, diplopia, blurred vision, gingival hyperplasia, nausea, vomiting, constipation, thrombocytopenia, leukopenia, agranulocytosis,etc. ${ }^{1}$

Simon craig has quoted that phenytoin is also capable of inducing seizures in severe cases of phenytoin intoxication. ${ }^{2}$

Stilman $\mathrm{N}$ et al had also quoted that in $\begin{aligned} & \text { Address for } \\ & \text { correspondence: }\end{aligned}$

some cases the seizure activity was attrib- Mr. Anup Jagarlamudi uted to phenytoin toxicity and the only factor that seemed to correlate with seizure activity was a serum phenytoin level over $30 \mathrm{mcg} / \mathrm{ml}^{3}$

Larry B. Mellick et al had quoted other adverse effects of phenytoin toxicity like: agitation/combativeness, sluggish pupil/ normal size, hyperreflexia, somnolent/ stuporous, vomiting, difficulty, eye fixation, babinski reflex, confusion/disorientation, hyporeflexia, widebae/staggering gait, dysarthria, tremor, ankle clonus, irregular respirations, absent corneal reflexes, opisthotonic posturing, etc. ${ }^{4}$
Submitted date : 12/07/2015 Accepted date : 13/01/2016

DOI: 10.5530/ijopp.9.1.12 M.Pharm,

Assistant Professor,

Department of Clinical Pharmacy Practice,

Pulla reddy Institue of Pharmacy, Domadugu village,

Near Annaram Air Force Academy, Jinnaram Mandal, Medak District,

Telengana-502313,INDIA.

Phone no: 91-7382670545

E-mail: anupjagarlamudi@

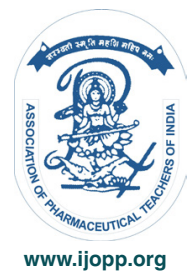


Suicidal behaviour/ideation has been warned by Dilantin manufactures. ${ }^{5}$

Lateral nystagmus may occur at levels above $20 \mathrm{mcg} / \mathrm{ml}$; ataxia usually occurs at levels above $30 \mathrm{mcg} / \mathrm{ml}$; significantly decreased mental capacity occurs at $40 \mathrm{mcg} / \mathrm{ml} .{ }^{1}$

Also many drugs, when administerd along with phenytoin, may increase the therapeutic effects of phenytoin. These drugs are: benzodiazepines, diazepam, valproic acid, etc. ${ }^{1}$

\section{CASE REPORT}

A female patient of age 24 years and weighing $45 \mathrm{kgs}$ was admitted to the hospital with 2 episodes of seizures. She is a known case of epileptic and is under regular medication. Upon admission her vitals were stable: with a pulse rate of 88 beats/minute and BP of $120 / 80 \mathrm{mmHg}$ and she was put on the following drugs: eptoin injection $200 \mathrm{mg}$ BD, lorazepam $2 \mathrm{mg}$ SOS, iv fluids NS, RL, DNS 1 unit each per day. On the next day valproic acid $200 \mathrm{mg}$ BD was substituted in place of lorazepam. By the evening of second day the patient started complaining of generalised weakness and also a fall in both heart rate and BP was noted by the duty medical officer (i.e. a fall of 14 beats/minute and 20/10 mmHg from base line). The EEG report had given a normal awake record. By night patient had started complaining of headache. In the morning of day 3 , her pulse rate was noted to be 88 beats/minute and $\mathrm{BP}$ was $90 / 60 \mathrm{mmHg}$. On day 4 the patient was put on oral phenytoin (i.e. a total of $300 \mathrm{mg} /$ day of oral phenytoin). In the midnight of day 5 the patient had 1 episode of generalised clonic tonic seizures and she was immediately administerd with $2 \mathrm{mg}$ of IV lorazepam stat. On day 6 around midday the patient had one more episode of seizure which lasted for 20 minutes and by the evening of that day the patient had developed ataxia, and was unable to hold her neck in place and also there was a notable drowsiness. The physician had added levitriacetam $500 \mathrm{mg}$ IV BD to the already existing prescription. Later that day, neurophysician visited the patient and opined that the patient is affected by Nystagmus, Truncal ataxia, Hyperventilation, Headache, fear feeling, and tremors of limbs and yet recommended to continue the same treatment. Also a psychiatrist had visited the patient on the same day and opined that the patient was under sleep, irritable, unhappy, weeping during the interview, suicidal thoughts, and worrying was also present and he had advised to administer antidepressant durgs: mirtazepine $7.5 \mathrm{mg}$ OD and escitalopram $5 \mathrm{mg}$ OD. On day 7 the patient had difficuly in holding neck, legs and was also unable to walk. Later on she was discharged on day 10 after the symptoms of ataxia had subsided.

\section{DISCUSSION}

Phenytoin is extensively used for rapid control of seizures. Phenytoin interacts with many anticonvulast drugs that belong to other classes when they are administerd concomitantly. A study on 25 phenytoinstable patients (300-400 mg/day) showed a nearly twofold increase in serum phenytoin concentrations, when patients also received either chlordiazepoxide or diazepam. ${ }^{6}$ Several other reports described increased serum phenytoin concentrations when receiving concomitant benzodiazepines (including chlordiazepoxide, clonazepam, and diazepam). ${ }^{7-10}$ Phenytoin also interacts with valproic acid and their use together should be with utmost care. Valproic acid displaces phenytoin from its binding sites i.e. proteins and hence, the metabolism of phenytoin is delayed, this interaction increases the therapeutic effects or toxic effects of phenytoin. Also, valproic acid increases the peak concentrations and AUC of lorazepam by $8 \%$ and $20 \%$ respectively when these two drugs are administered together. ${ }^{11}$ Levitiracetam and mirtazepine when administered concomitantly, impairs motor functions.

\section{CONCLUSION}

Although phenytoin is being extensively used for controlling seizures, it should be remembered that it is also a narrow therapeutic index drug and also has got some severe drug-drug interactions with a wide variety of agents and the possible outcomes of such ineraction is either increased serum phenytoin levels or decreased serum phenytoin levels, both of these outcomes are undesirable as increased serum phenytoin levels causes seroius adverse effects and decreased serum phenytoin levels will not control the seizure activity. Hence, TDM (therapeutic drug monitoring) is recommended whenever phenytoin is choosen for controlling seizures.

In the above case, inclusion of TDM in the pharmaceutical care plan would have prevented the adverse effects of phenytoin, as it is clear from the discussion part that in the above case there is a clear evidence of increased serum concentrations of phenytoin due to many drug-drug interactions. Also, data suggests that there are other classes of anticonvulsants (eg: valproic acid, lorazepam, etc.) that are as effective as phenytoin and hence withdrawal of phenytoin is another option of overcoming such serious adverse effects due to drugdrug interactions. 


\section{ABBREVIATION USED}

GTCS: $\quad$ Generalised tonic clonic seizures

AUC: $\quad$ Area under the curve

TDM: Therapeuitc drug monitoring

\section{ACKNOWLEDGEMENTS}

The Author acknowledges the support given by the Department of Clinical Pharmacy Practice, Pullareddy Institute of Pharmacy and also the Administration of Ram Dev Rao Hospital, Kukkatpally, Hyderabad.

\section{CONFLICT OF INTEREST}

The authors have no conflicts of interest.

\section{REFERENCES}

1. Pharmacist's Drug Handbook: ASHP, Maryland; Springhouse Corporation, Pennsylvania; 2001. phenytoin, phenytoin sodium, phenytoin sodium (extended); 904

2. Simon $\mathrm{C}$, Phenytoin poisoning; Perspectives in Intoxications. Neurocritical care. 2005;3(2):161-70.

3. Stilman N, Masdeu JC. Incidence of seizures with phenytoin neurotoxicity. Neurology. 1985;35(12):1769-72.

4. Mellick LB, Morgan JA, Garya AM. Presentations of Acute Phenytoin Overdose. American Journal of Emergency Medicine. 1989;7(1):61-7.

5. Dilantin [Package insert]. NY 10017. Parke-Davis, division of Pfizer Inc, NY; 2009.

6. Vajda FJ, Prineas RJ, Lovell RR. Interaction Between Phenytoin and the Benzodiazepines. BMJ. 1971;1(5744):346.

7. Shuttleworth E, Wise G, Paulson G. Choreoathetosis and Diphenylhydantoin Intoxication. JAMA. 1974;230(8):1170-1.

8. Windorfer P. Drug Interactions During Anticonvulsive Therapy. Int J Clin Pharmacol. 1977;8(1):29-41.

9. Kutt H, McDowell FJ. Management of Epilepsy With Diphenylhydantoin Sodium. JAMA. 1968;203(11):969-72.

10. Murphy A, Wilbur K. Phenytoin-Diazepam Interaction. Ann Pharmacother. 2003;37(5):659-63.

11. Available from: http://www.clinicalpharmacology.com/Forms/Reports/intereport. aspx?gpcid $=893,931,1906 \& \mathrm{l}=0$ 